\title{
INDEPENDENT EVENTS AND INDEPENDENT EXPERIMENTS
}

\author{
YULIY M. BARYSHNIKOV AND BENNETT EISENBERG \\ (Communicated by George C. Papanicolaou)
}

\begin{abstract}
It is shown that independent events in a probability space with equally likely outcomes are isomorphic to events coming from independent experiments each with equally likely outcomes.
\end{abstract}

\section{INTRODUCTION}

Let $\Omega$ be a finite set with uniform probability distribution $P$. Denote the cardinality of $\Omega$ by $|\Omega|=\prod_{j=1}^{n} p_{j}$, where the $p_{j}$ 's are prime numbers greater than one, which are not necessarily distinct. Eisenberg and Ghosh [1] show that there does not exist a collection of more than $n$ nontrivial mutually independent events in $\Omega$, where $n$ is the number of prime factors of $|\Omega|$. An event is called trivial if it has probability 0 or 1 . The proof is complicated by the fact that it uses the number of times that each distinct prime number occurs in the factorization of $|\Omega|$. In this article we give a more unified proof of the result of Eisenberg and Ghosh.

Next consider the product space $\Omega_{1} \times \cdots \times \Omega_{n}$ with the uniform probability distribution where $\left|\Omega_{j}\right|=p_{j}$. The $\Omega_{j}$ 's can be interpreted as spaces of outcomes of independent experiments, each with equally likely outcomes. If $A_{j}$ is a nontrivial cylinder set based on $\Omega_{j}$ for $j=1, \ldots, n$, then $A_{1}, \ldots, A_{n}$ form a collection of $n$ nontrivial mutually independent events in $\Omega_{1} \times \cdots \times \Omega_{n}$. These are the most fundamental types of independent events since they are events related to independent experiments. There is a 1-1 map from this product space to $\Omega$, and under this isomorphism the images of the $A_{j}$ 's are nontrivial mutually independent events in $\Omega$. In this article we show that any set of nontrivial mutually independent events in $\Omega$ must be of this form; that is, mutually independent events in $\Omega$ must be isomorphic to events from independent experiments with equally likely outcomes.

Received by the editors September 25, 1991.

1991 Mathematics Subject Classification. Primary 60C05; Secondary 60A99.

Key words and phrases. Independent events, independent experiments, equally likely outcomes, prime factorization.

The first author's research was supported by the Alexander von Humboldt Foundation, Germany.

The second author's research was done while visiting the University of Osnabruck. 


\section{INDEPENDENT EVENTS IN A SPACE OF $N$ EQUALLY LIKELY OUTCOMES}

The main results are corollaries to the following lemma. The notation of the lemma is used throughout this section.

Lemma. Let $\Omega$ be a probability space with $N$ equally likely outcomes. Assume that $A_{1}, \ldots, A_{m}$ are nontrivial mutually independent events in $\Omega$. Let $\mathfrak{F}_{0}=$ $\{\phi, \Omega\}$ and let $\mathfrak{F}_{j}$ be the field generated by $\left\{A_{1}, \ldots, A_{j}\right\}$ for $j=1, \ldots, m$. Let $N_{j}$ be the greatest common divisor of the cardinalities of the sets in $\mathfrak{F}_{j}$. Then

(1) $N_{0}=N$ and $N_{j}$ is a proper divisor of $N_{j-1}$ for $j=1, \ldots, m$ and

(2) $P\left(A_{j}\right)=h_{j} N_{j} / N_{j-1}$ for an integer $h_{j}$ with $1 \leq h_{j}<N_{j-1} / N_{j}$.

Proof. Since $\mathfrak{F}_{j-1} \subset \mathfrak{F}_{j}$, it follows that $N_{j}$ divides the cardinality of every set in $\mathfrak{F}_{j-1}$, and hence $N_{j}$ divides $N_{j-1}$. We must show that it is a proper divisor, i.e., $N_{j}<N_{j-1}$.

From the definition of $N_{j-1}$, it follows that there exists a representation $N_{j-1}=\sum_{\alpha} k_{\alpha}\left|B_{\alpha}\right|$, where the $k_{\alpha}$ 's are integers and the $B_{\alpha}$ 's are sets in $\mathfrak{F}_{j-1}$. Form the sum $S=\sum k_{\alpha}\left|B_{\alpha} A_{j}\right|$. Since $A_{j}$ is independent of $\mathfrak{F}_{j-1}$, it follows that $\left|B_{\alpha} A_{j}\right|=P\left(B_{\alpha} A_{j}\right) N=P\left(B_{\alpha}\right) P\left(A_{j}\right) N=\left|B_{\alpha}\right| P\left(A_{j}\right)$. Thus $S=$ $\sum k_{\alpha}\left|B_{\alpha}\right| P\left(A_{j}\right)=N_{j-1} P\left(A_{j}\right)$. Since $S$ is a sum of integral multiples of the cardinalities of sets in $\mathfrak{F}_{j}$, it must be divisible by $N_{j}$. Thus $N_{j-1} P\left(A_{j}\right)=h_{j} N_{j}$ for some integer $h_{j}$. Since $0<P\left(A_{j}\right)<1$, it follows that $1 \leq h_{j}<N_{j-1} / N_{j}$. Hence $N_{j}=P\left(A_{j}\right) N_{j-1} / h_{j}<N_{j-1}$. This proves (1).

Furthermore, $P\left(A_{j}\right)=h_{j} N_{j} / N_{j-1}$, which gives (2).

Theorem 1. If $A_{1}, \ldots, A_{m}$ are nontrivial mutually independent events in $\Omega$ then $m \leq n$, where $n$ is the number of prime factors of $\Omega$.

Proof. Suppose $m>n$. From the lemma, $N_{1}$ is a proper divisor of $N$. Hence $N_{1}$ has at most $n-1$ prime factors. Continuing in this way, $N_{m}$ has at most $n-m$ prime factors. Thus $N_{n}=1$, which has no proper divisors. But the lemma implies that $N_{n+1}$ must be a proper divisor of $N_{n}$. This is a contradiction.

Theorem 2. If $A_{1}, \ldots, A_{m}$ are nontrivial mutually independent events in $\Omega$, then there exists an isomorphism from $\Omega$ to $\Omega^{\prime}=\Omega_{1} \times \cdots \times \Omega_{m} \times \Omega_{m+1}$, where $\left|\Omega_{j}\right|=N_{j-1} / N_{j}$ for $j=1, \ldots, m$ and $\left|\Omega_{m+1}\right|=N_{m}$, such that $A_{j}$ gets mapped to a cylinder set based in $\Omega_{j}$ for $j=1, \ldots, m$.

Proof. According to the lemma, $P\left(A_{j}\right)=h_{j} N_{j} / N_{j-1}$ with $1 \leq h_{j}<N_{j-1} / N_{j}$. Choose a set of $h_{j}$ points in $\Omega_{j}$ and let $A_{j}^{\prime}$ be the cylinder set based on this set. Then if all points in $\Omega^{\prime}$ are equally likely, we have that $P\left(A_{j}\right)=$ $P\left(A_{j}^{\prime}\right)$. Due to the independence of $A_{1}, \ldots, A_{m}$ in $\Omega$ and $A_{1}^{\prime}, \ldots, A_{m}^{\prime}$ in $\Omega^{\prime}$, we have that each atom in the field generated by $A_{1}, \ldots, A_{m}$ has the same probability as the corresponding atom in the field generated by $A_{1}^{\prime}, \ldots, A_{m}^{\prime}$. Since $|\Omega|=\left|\Omega^{\prime}\right|$, these atoms must have the same number of points as well. By choosing arbitrary 1-1 maps between points in the corresponding atoms, we get the desired isomorphism.

Corollary. If $A_{1}, \ldots, A_{n}$ are nontrivial mutually independent events in $\Omega$, where $n$ is the number of prime factors $|\Omega|$, then one can choose $\Omega^{\prime}=\Omega_{1}$ $\times \cdots \times \Omega_{n}$ in Theorem 2 . 
Proof. As in the proof of Theorem 1 we have that $N_{n}=1$ so that the factor $\Omega_{n+1}$ is not needed in order that $|\Omega|=\left|\Omega^{\prime}\right|$. The proof of Theorem 2 then applies to complete the proof.

Example. Let $\Omega=\{1,2,3,4,5,6\}, A_{1}=\{2,4,6\}$, and $A_{2}=\{1,2,3,4\}$. Then $A_{1}$ and $A_{2}$ are independent events. $N=N_{0}=6, N_{1}=3$, and $N_{2}=1$. $N_{0} / N_{1}=2$ and $N_{1} / N_{2}=3$. Let $\Omega_{1}=\{1,2\}$ and $\Omega_{2}=\{1,2,3\}$. We may then choose $A_{1}^{\prime}=\{(1,1),(1,2),(1,3)\}$ and $A_{2}^{\prime}=\{(1,1),(2,1),(1,2)$, $(2,2)\}$ so that $A_{1}^{\prime}$ represents the event " 1 on experiment 1 " and $A_{2}^{\prime}$ represents the event " 1 or 2 on experiment 2." The isomorphism is then any 1-1 map that takes $A_{1} A_{2}=\{2,4\}$ to $A_{1}^{\prime} A_{2}^{\prime}=\{(1,1),(1,2)\}$, that takes $A_{1} A_{2}^{c}=\{6\}$ to $A_{1}^{\prime} A_{2}^{\prime c}=\{(1,3)\}$, that takes $A_{1}^{c} A_{2}=\{1,3\}$ to $A_{1}^{\prime c} A_{2}^{\prime}=\{(2,1),(2,2)\}$, and takes $A_{1}^{c} A_{2}^{c}=\{5\}$ to $A_{1}^{\prime c} A_{2}^{c c}=\{(2,3)\}$. One way to achieve this is $1 \rightarrow(2,1)$, $2 \rightarrow(1,1), 3 \rightarrow(2,2), 4 \rightarrow(1,2), 5 \rightarrow(2,3)$, and $6 \rightarrow(1,3)$.

In general these conclusions do not apply to pairwise independent events. For example, Eisenberg and Ghosh show that if $|\Omega|=k^{2}$ for any $k \geq 2$, then there exists a collection of $k+1$ nontrivial pairwise independent events in $\Omega$. For example, if $|\Omega|=25$ then there is a collection of six nontrivial pairwise independent events. On the other hand, there can be no more than two nontrivial mutually independent events in this case. Theorem 1 does extend to pairwise independent events, however, if the prime factors of $|\Omega|$ are all distinct. This result is also proved in the Eisenberg and Ghosh article.

\section{REFERENCES}

1. B. Eisenberg and B. K. Ghosh, Independent events in a discrete uniform probability space, Amer. Statist. 41 (1987), 52-56.

Department of Mathematics, University of Osnabruck, Osnabruck, Germany E-mail address: yuliy@chryseis.mathematik.uni-osnabrueck.de

Department of Mathematics, Lehigh University, Bethlehem, Pennsylvania 18015 E-mail address: BE01@Lehigh.EDU 